\title{
SURGICAL TREATMENT OF PERIODONTAL INTRABONY DEFECTS WITH CALCIUM SULPHATE IN COMBINATION WITH BETA TRICALCIUM PHOSPHATE - A 12-MONTH RETROSPECTIVE CLINICAL EVALUATION
}

\author{
Sujith Sukumar', Ivo Dřizhal ${ }^{1}$, Josef Bukač², Vladimíra Paulusová1, Shriharsha Pilathadka ${ }^{1}$
}

Charles University in Prague, Faculty of Medicine and University Hospital Hradec Králové, Czech Republic: Department of Dentistry ${ }^{1}$, Department of Medical Biophysics ${ }^{2}$

\begin{abstract}
Summary: Alloplastic bone graft materials are widely been used in combination with barrier membranes to achieve guided tissue regeneration in the treatment of periodontal intrabony defects. This study was designed to evaluate the clinical outcome of a composite material, beta tricalcium phosphate in combination with calcium sulphate in the treatment of periodontal intrabony defects. The combination of these materials is believed to aid in guided tissue regeneration owing to their properties. Thirty nine intrabony defects in 21 patients were treated with Fortoss ${ }^{\circledR}$ Vital (Biocomposites, Staffordshire, UK). Clinical parameters were evaluated including changes in probing depth, clinical attachment level/loss and gingival recession at baseline and one year postoperatively. The mean differences in measurements between the baseline and one year postoperatively are a reduction of $1.98 \pm 1.16 \mathrm{~mm}(\mathrm{p}=0.000)$ in case of probing depth and a gain of $1.68 \pm 1.12 \mathrm{~mm}$ $(p=0.000)$ in clinical attachment level and an increase of $0.31 \pm 0.67 \mathrm{~mm}(\mathrm{p}=0.009)$ in gingival recession measurements. The study results show that the treatment with a combination of beta tricalcium phosphate and calcium sulphate led to a significantly favorable clinical improvement in periodontal intrabony defects one year postoperatively.
\end{abstract}

Key words: Beta tricalcium phosphate; Calcium sulphate; Periodontal regeneration; Intrabony defect; Bone graft

\section{Introduction}

Contemporary periodontal therapy is directed towards controlling the infection and regenerating lost supporting structures. Infection control can be achieved by proper initial phase periodontal therapy including through scaling and root planing, maintenance and antimicrobial therapy. The ultimate goal of periodontal therapy is regeneration of periodontal tissues. Periodontal regeneration refers to the restoration of supporting tissues of the teeth, which is the alveolar bone, cementum, and periodontal ligament to their original healthy levels before tissue destruction caused by periodontal pathogens has occurred. Histological examination of the newly formed tissues has to be done to establish that the regeneration has occurred $(5,11,27)$. Currently, osseous grafting and guided tissue regeneration (GTR) are the two techniques with the most histological documentation of periodontal regeneration (15). In most of the recent studies comparing GTR to conventional therapy or open flap debridement, GTR was found to have a greater effect on probing measures of periodontal treatment than open flap debridement, including improved attachment gain, reduced pocket depth and less increase in gingival recession $(20,21)$. Needleman et al. reported a greater mean attach- ment gain of $1.22 \mathrm{~mm}$ when comparing GTR to open flap debridement (21).

The interest in bone replacement grafts has emerged from the desire to fill an intrabony or furcation defect rather than radically resect surrounding intact bone tissue. It is assumed that the application of bone grafts would potentially manipulate the biological response into a regenerative rather than a predominantly reparative pattern of periodontal healing (12).

Guided tissue regeneration (GTR) describes the procedures designed to manipulate the cells that repopulate the wound healing site so as to ensure that this repopulation leads to regeneration of the periodontal tissues. GTR aims to isolate the periradicular bone wound from the rest of the tissues (epithelial, connective and periosteal). This will allow the cells from the periodontal ligament to repopulate the blood coagulum that forms between the alveolar bone and root surface. The isolation of the wound is achieved using a physical barrier like a membrane $(7,9,10,18,20$, 21). There are different types of barrier membranes, both resorbable and non-resorbable. A better clinical outcome is anticipated when bone grafts are used in combination with GTR. This led to the widespread use of the combination technique. Different graft materials were used with diffe- 
rent types of membranes in an attempt to achieve the best possible results. However, research on the value of using a combined technique is not universal.

Alloplasts are synthetic, inorganic, biocompatible bone substitutes that primarily functions as defect fillers. Alloplasts can aid in bone regeneration by a process called osteoconduction. Beta tricalcium phosphate is one of the earliest calcium compounds to be used as a bone graft substitute. Structurally porous beta tricalcium phosphate has a compressive strength and tensile strength similar to that of cancellous bone. It undergoes resorption over a 6-18 month period. Unfortunately, the replacement of beta tricalcium phosphate by the bone does not occur in an equitable way. That is, there is always less bone volume produced than the volume of the graft material resorbed. For this reason, the clinical use of beta tricalcium phosphate has been rather as an adjunctive with other less resorbable bone graft substitutes or as an expander for autogenous bone graft $(17,28)$.

Another calcium compound that is used as a bone void filler is calcium sulphate. It has a compressive strength greater than that of cancellous bone. It can act as a barrier membrane as well, which makes it ideal as an adjunct with other graft materials (23). The goal of the current study was retrospective evaluation of the clinical outcome of a composite material, beta tricalcium phosphate in combination with calcium sulphate, in the treatment of human periodontal intrabony defects.

\section{Methods}

\section{Set of patients}

In this study 21 patients with chronic periodontitis treated with the composite material (Fortoss ${ }^{\circledR}$ Vital, Biocomposites, Staffordshire, UK) were evaluated. These patients were in good general health. They were treated in the department of Dentistry, Faculty of Medicine and Faculty Hospital in Hradec Králové, Czech Republic. They were aged from 21 to 53 years with a mean age of 43 at the time of the surgery. There were 8 males and 13 females, out of which 7 were medium smokers, smoking up to 5 cigarettes a day.

Subject inclusion was based on the presence of at least one tooth with a probing depth (PD) of more than or equal to $(\geq) 5 \mathrm{~mm}$ and radiographic evidence of intrabony defect after initial periodontal therapy. The exclusion criteria consisted of patients with systemic diseases, pregnant and/or lactating women, and taking any drug known to affect the number or function of platelets in the past 3 months. Teeth had to be vital or properly treated with root canal therapy. Signed consent form before surgery was obtained from each patient.

\section{Study design and clinical procedures}

All patients underwent initial therapy, consisting of oral hygiene instruction, full-mouth scaling and root planing, elimination of local plaque-retaining factors and restorations and occlusal adjustments when indicated. From all the pa- tients a total of 39 periodontal intrabony defects were included in this study. These were either 2 or 3 walled defects with 25 of those located around the anterior teeth and 7 each around the premolars and molars. All the defects were treated by one clinician, using a standardized procedure. Clinical parameters like probing depth (PD), gingival recession (GR), clinical attachment level/loss (CAL), presence/absence of dental plaque and bleeding on probing (BOP) were recorded just before the surgery (baseline) and at 12 months postoperatively. Radiographs were also made before the surgery and at 12 months postoperatively. The clinical parameters at the baseline and at 12 months postoperatively were compared and evaluated statistically to procure the study outcome. Radiographs were also compared to support the clinical outcome.

The surgical treatment phase was initiated only if the subject had a papilla bleeding index (PBI) sum score (bleeding number) of $\leq 15$. After achieving sufficient local anesthesia, a full-thickness mucoperiosteal flap was elevated using a crevicular incision on the facial and lingual surfaces of each tooth, segment or area involved. In the upper anterior region a papilla preservation incision was made in the interdental area. Vertical release incisions were used as necessary. After the elevation of the flap, a thorough root surface debridement was done using Gracey or universal curettes (Hu-Friedy, Chicago, IL, USA). All granulomatous tissue were removed from the osseous defects and rinsed with saline. Root surface conditioning was done using 2.5 $\%$ tetracycline HCL for 2-3 minutes followed by flushing with saline. Fortoss ${ }^{\circledR}$ Vital powder was mixed with the liquid supplied along with it in to a gritty mouldable paste and applied it in layers using a sterile instrument. The graft material was firmly pressed into the site using finger pressure over sterile gauze. The defects were over-packed to allow for any settling of the mixture. Any excessive blood was removed from the site by using damp sterile gauze. Then the gauze was held on the graft for a few seconds. The mucoperiosteal flaps were approximated and sutured using resorbable sutures (Safil ${ }^{\circledR}$, Braun, Tuttlingen, Germany).

The patients were given post-operative instructions including rinsing with Listerine ${ }^{\circledR}$ (Johnson \& Johnson, Maidenhead, UK) mouth rinse for 2 weeks. Antibiotics (Amoxicillin $250 \mathrm{mg}$ with clavulanic acid $125 \mathrm{mg}$ or clarithromycin $500 \mathrm{mg}$ ) were prescribed to the patients for 7-14 days depending on the size and extend of the defects. The sutures were removed after 2 weeks and the surgical sites were cleansed gently with $3 \%$ hydrogen peroxide using a cotton swab. The patients were scheduled for recall visits at 3,6 and 12 months postoperatively. Oral hygiene was evaluated and supragingival prophylaxis was carried out at each recall visit. Nearly all the patients followed the regime except three.

\section{Clinical parameters}

The clinical measurements were performed by 2 examiners randomly. Both the examiners recorded similar mea- 
surements during a 2 year trial period of cross-checking. Clinical parameters were recorded before the surgery (baseline) and at 12 months postoperatively. These included probing depth (PD), gingival recession (GR), clinical attachment level/loss (CAL), presence/absence of dental plaque on the mesial and distal tooth surfaces, bleeding on probing (BOP) and mobility. The measurements were done using a Williams calibrated periodontal probe (Williams color coded, Hu-Friedy, Chicago, IL, USA) at the buccal, lingual, mesial and distal surfaces on all teeth involved and the highest value for each surface is quoted.

\section{Radiographs}

Intraoral periapical radiographs were taken at the baseline and at 12 months postoperatively. The radiographs were used only for the detection of bone fill in the defects and not for the measurements as the method employed was not standardized. Post-operative radiographs were compared to the ones at the baseline in order to evaluate the bone fill and also to compare that with the clinical parameters.

\section{Statistical analysis}

Comparisons between baseline and one year data were made using a paired t-test and Fisher's exact test. Mean differences in the PD, GR and CAL were calculated on individual surfaces and also on the whole tooth. All the surfaces of an involved tooth were taken into account irres-

Tab. 1: Total PD measurements $\geq 5 \mathrm{~mm}$ : at baseline and 12 months postoperatively.

\begin{tabular}{|l|c|c|c|c|}
\hline & V & M & O & D \\
\hline Baseline & 7 & 29 & 15 & 18 \\
\hline 12 months & 0 & 4 & 3 & 2 \\
\hline
\end{tabular}

pective of the presence/absence of $\mathrm{PD} \geq 5 \mathrm{~mm}$. This was done to assess the outcome of surgery on the non-involved sites of the involved tooth as well. Data were expressed as mean \pm standard deviations. The level of significance was set at 0.05 .

\section{Results}

Clinically, the graft material used was easy to handle, strongly adherent, packed well into defects, appeared to harden as a solid in a few minutes and appeared to be biocompatible. Wound healing was uneventful. No patients reported a significant postoperative pain during the first week. Except three, all the other patients returned for all scheduled recall visits. All of them reported at 12 months postoperatively.

A decrease in probing depths was noticed in 18 patients out of the total 21 . In the other three, two of them did not respect the scheduled recall visits. There was no change in $\mathrm{PD}$ in two and an increase in PD in one patient. The number of BOP positive sites was reduced from 56 at baseline to 26 at 12 months postoperatively. But the number of sites with presence of supragingival plaque deposits got increased from 8 to 25 during the same interval.

Table 1 shows the differences between deep probing depth measurements at the baseline and at 12 months postoperatively. There were a total of 69 PD measurements $\geq 5$ $\mathrm{mm}$ in those 39 involved teeth at the baseline. A 12 month

Tab. 2: Total CAL measurements $\geq 5 \mathrm{~mm}$ : at baseline and 12 months postoperatively.

\begin{tabular}{|l|c|c|c|c|}
\hline & V & M & O & D \\
\hline Baseline & 11 & 30 & 22 & 25 \\
\hline 12 months & 8 & 22 & 12 & 19 \\
\hline
\end{tabular}

Tab. 3: Mean differences in gingival recession (GR) measurements before and 12-months after the surgery.

\begin{tabular}{|c|c|c|c|c|}
\hline \multicolumn{5}{|c|}{ GR (increase = "+", decrease = "“) } \\
\hline $\mathrm{V}$ & $\mathrm{M}$ & $\mathrm{O}$ & $\mathrm{D}$ & Average \\
\hline$+0.54(-1$ to 3$)$ & $+0.18(-3$ to 3$)$ & $+0.38(-2$ to 2$)$ & $+0.26(-3$ to 3$)$ & +0.31 \\
SD: \pm 0.98 & SD: \pm 1.18 & SD: \pm 0.97 & SD: \pm 1.52 & SD: \pm 0.67 \\
\hline
\end{tabular}

Tab. 4: Mean differences in periodontal probing depth (PD) measurements before and 12-months after the surgery.

\begin{tabular}{|c|c|c|c|c|}
\hline \multicolumn{5}{|c|}{ PD (increase = "+", decrease ="“) } \\
\hline $\mathrm{V}$ & $\mathrm{M}$ & $\mathrm{O}$ & $\mathrm{D}$ & Average \\
\hline $0.89(-7$ to 3$)$ & $-3.07(-9$ to 5$)$ & $-1.53(-6$ to 2$)-$ & $2.37(-9$ to 1$)$ & -1.98 \\
SD: \pm 1.91 & SD: \pm 2.76 & SD: \pm 1.96 & SD: \pm 2.28 & SD: \pm 1.16 \\
\hline
\end{tabular}

Tab. 5: Mean difference in clinical attachment level (CAL) measurements before and 12-months after the surgery.

\begin{tabular}{|c|c|c|c|c|}
\hline \multicolumn{5}{|c|}{ CAL (Gain= “+”, Loss= “") } \\
\hline $\mathrm{V}$ & $\mathrm{M}$ & $\mathrm{O}$ & $\mathrm{D}$ & Average \\
\hline$+0.36(-2$ to 7$)$ & $+2.92(-4$ to 9$)$ & $+1.14(-2$ to 5$)$ & $+2.11(-3$ to 8$)$ & $+\mathbf{1 . 6 8}$ \\
SD: \pm 1.89 & SD: \pm 2.89 & SD: \pm 2.08 & SD: \pm 2.59 & SD: \pm 1.12 \\
\hline
\end{tabular}

$\mathrm{V}=$ vestibular/buccal, $\mathrm{M}=$ mesial, $\mathrm{O}=$ oral/lingual, $\mathrm{D}=$ distal, $\mathrm{SD}=$ standard deviation 
postoperative recall check showed a significant decrease in this number to 9 .

Table 2 shows how the CAL measurements fared during the same period. Total CAL measurements of $\geq 5 \mathrm{~m}$ at the baseline was 88 which got reduced to 61 at 12 months postoperatively.

The mean differences in measurements between the baseline and one year postoperatively were a reduction of $1.98 \pm 1.16 \mathrm{~mm}(\mathrm{p}=0.000)$ in case of $\mathrm{PD}$, a gain of $1.68 \pm 1.12$ $\mathrm{mm}(\mathrm{p}=0.000)$ in CAL and an increase of $0.31 \pm 0.67 \mathrm{~mm}$ $(p=0.009)$ in GR. These findings are illustrated in tables 3, 4 and 5. Table 3 shows that the mean increase in GR was much lower in the mesial and distal aspects of teeth. Also, the maximum increase in GR at 12 month postoperatively was $3 \mathrm{~mm}$ and the maximum decrease achieved was also 3 $\mathrm{mm}$.

In table 4 the changes in PD is shown. There was a significant mean reduction in the proximal aspects of the teeth with $9 \mathrm{~mm}$ being the maximum reduction in PD and $5 \mathrm{~mm}$ the maximum increase. Table 5 shows the mean gain of CAL, which was much higher in the mesial and distal aspects.

Intraoral periapical radiographs showed bone fill in the defects in patients where PD got reduced after the surgical treatment. There were no significant differences between smokers and non-smokers $(p=1.000)$ and presence or absence of plaque and BOP. In one patient where an increase in PD and CAL were noticed 12-months after the surgery, a combination of different factors like smoking, bad oral hygiene and non-compliance with the follow-up schedule during the maintenance phase after surgery, were found. The patient came for the follow-up only after one year.

\section{Discussion}

Bone grafting is now a well-recognized mode of treatment of periodontal intrabony defects, especially when used along with barrier membranes. Various types of bone grafts and also their combinations are used with varying degrees of success. The use of a composite graft containing betatricalcium phosphate and calcium sulphate was described in only a few reports and studies $(24,29,30)$. In those reports and studies it was found that the use of this particular graft provided good results. The study done on the iliac crest of dogs by Podaropoulos et al. in 2009 revealed that the mean percentage of new bone regeneration after 4 months by histological evaluation and morphometric analysis was $49.38 \%$. Structurally porous beta tricalcium phosphate has a compressive strength and tensile strength similar to that of cancellous bone. It undergoes resorption over a 6-18 month period. Calcium sulphate has a compressive strength greater than that of cancellous bone. It can act as a barrier membrane as well, which makes it ideal for using as an adjunct with other graft materials. It requires only 5-7 weeks for complete resorption $(3,28)$.

Fortoss ${ }^{\circledR}$ Vital which is a combination of betatricalcium phosphate and calcium sulphate is being used in the treat- ment of periodontal intrabony defects in our department since 2003. The main reasons for the choice of this material over the conventional membrane and graft technique to achieve periodontal regeneration are non-requirement of a membrane, reduced surgical time, lesser cost and the ease and potential to treat periodontal intrabony defects spanning more than two teeth.

Results from our present investigation showed that the graft material used was effective in significantly improving the clinical parameters at one year after surgery. Ideally, a comparative study with open flap debridement and/or using a different bone graft material in treating comparable defect pairs would have been more significant to highlight the outcome of treatment using Fortoss ${ }^{\circledR}$ Vital. The amount of $\mathrm{PD}$ reduction was found to be greater in deeper defects. In some cases, this reduction was up to $9 \mathrm{~mm}$. PD reduction was achieved in 18 of the total 21 patients; there was no change in 2 and an increase in 1 of them. The local factors and the non-compliance of the patient probably would have resulted in the undesired result. After one year, the number of sites with bleeding on probing was reduced to almost half. But the number of proximal sites (mesial and distal) with supragingival plaque deposits was increased in the same period indicating that there was no correlation between both factors in our study group.

Several studies were done to evaluate the effectiveness of calcium sulphate and also of beta tricalcium phosphate in combination with other materials resulting in good clinical outcomes. A study by Harris RJ in 2004 evaluating a composite bone graft (demineralized freeze-dried bone allograft, calcium sulphate, tetracycline and porous hydroxyapatite) and calcium sulphate barrier showed a mean decrease of $4.7 \mathrm{~mm}$ of PD, $3.7 \mathrm{~mm}$ of CAL and a mean increase of $1.0 \mathrm{~mm}$ of GR at 4-6 months postoperatively (16). In another study by Paolantonio $M$ et al. in 2008 using calcium sulphate barrier implant and barrier revealed a mean decrease of $4.4 \mathrm{~mm}$ of PD, $2.7 \mathrm{~mm}$ of CAL and a mean increase of $1.6 \mathrm{~mm}$ of GR at 12 months postoperatively (22). In a study published in 2008 by Döri F, at 1 year after therapy, the sites treated with platelet rich plasma and $\beta$-TCP using GTR showed a reduction in mean PD from 9.1 $\pm 0.6 \mathrm{~mm}$ to $3.3 \pm 0.5 \mathrm{~mm}(\mathrm{P}<0.001)$ and a change in mean CAL from $10.1 \pm 1.3 \mathrm{~mm}$ to $5.7 \pm 1.1 \mathrm{~mm}$ (8). Most of these studies used clinical measurements along with standardized radiographs for comparison. Unlike the present study, all these studies have considered only the affected area around the tooth, where the pocket depths were deeper, which may influence the results.

Clinical parameters for the assessment of clinical outcome variables, such as probing depths, attachment level and gingival recession, have varied between studies, particularly with regard to the use of automatic or pressure sensitive or conventional probes and the use of a stent as a reference point (25). The key element is the consistency of the assessment throughout the study. In the present study, an occlusal stent was not fabricated; the cement enamel 
junction and the free gingival margin served as reference points. Manual probes were used to measure the variables. The ability of a probe to penetrate into a periodontal pocket is related to several factors like the probing force, diameter of the probe and the gingival tissue tone $(1,19,26)$. In the present study, Williams color-coded probe (Hu-Friedy, Chicago, IL, USA) was used throughout in order to ensure the consistency in probe diameter. The clinical measurements were performed by 2 examiners randomly. Both the examiners recorded similar measurements during a 2 year trial period of cross-checking which ensured the similarity in probing force and method. Gingival tissue consistency may be modified after the placement of graft material in to the defect, which in turn can impede penetration of the periodontal probe causing false positive results (4).

Unlike usual studies, we have considered the unaffected sides of the tooth as well. We have done this as the surgical wound included all the sides of the tooth. But this has affected the outcome, with a lesser than expected gain of attachment even though the reduction in deep pocket depths are much more significant. The shortcomings of our study could be a small patient group, absence of radiographic analysis or surgical re-entry to establish the bone fill/regeneration and the non-usage of stents during clinical measurements.

Conventional therapy is capable of controlling periodontal disease. Scaling and root planning allow the reduction in pocket depth mainly by new connective tissue/epithelial attachment; with a probable gain in clinical attachment level. Periodontal literature is sated with studies showing the treatment of periodontitis by scaling and root planing results in reductions of probing depths $(2,6,13,14)$. The decrease in probing depth is caused partly by the shrinkage of the pocket soft tissue wall manifested as recession of the gingival margin which results from a decrease in soft tissue inflammation; and partly from the gain in clinical attachment $(2,6,13,14)$. In a thorough evidence-based review published in 1996, Cobb calculated the mean probing depth reduction and gain of clinical attachment that can be achieved with root planing at sites that initially were 4 to 6 $\mathrm{mm}$ in depth and $7 \mathrm{~mm}$ or greater in depth. He reported mean pocket depth reductions of $1.29 \mathrm{~mm}$ and $2.16 \mathrm{~mm}$, respectively, and mean gains of clinical attachment of 0.55 $\mathrm{mm}$ and $1.29 \mathrm{~mm}$, respectively (6).

The regenerative procedures are performed after the conventional scaling and root planing to attain further improvement of the tissues destroyed by periodontitis. These procedures can promote further bone fill, thus improving the supporting structures thereby possibly improving the long-term prognosis of the tooth.

\section{Conclusion}

The treatment with a synthetic bone graft containing a combination of betatricalcium phosphate and calcium sulphate led to a significantly favorable clinical improve- ment in periodontal intrabony defects one year after the surgery. A longer term evaluation and further studies are necessary to completely ascertain the effectiveness of this material and procedure, and a larger sample size is needed. Also, standardized radiographic evaluation or a surgical reentry is recommended for confirmation of the clinical results.

\section{Acknowledgement}

This paper was supported by the research grant FN HK MZO 00179906.

\section{References}

1. Armitage GC, Svanberg GK, Loe H. Microscopic evaluation of clinical measurements of connective tissue attachment levels. J Clin Periodontol 1977; 4: 173-190.

2. Badersten A, Nilveus R, Egelberg J. Effect of nonsurgical periodontal therapy. I: moderately advanced periodontitis. J Clin Periodontol. 1981; 8: 57-72.

3. Bell WH. Resorption rates of bone and bone substitutes. Oral Surg. 1964; 17: 650-7.

4. Camargo PM, Lekovic V, Weinlaender M, et al. A controlled re-entry study on the effectiveness of bovine porous bone mineral used in combination with a collagen membrane of porcine origin in the treatment of intrabony defects in humans. J Clin Periodontol 2000; 27: 889-896.

5. Caton J, Nyman S, Zander H. Histometric evaluation of periodontal surgery: II. Connective tissue attachment levels after four regenerative procedures. J Clin Periodontol 1980; 7: 224-231.

6. Cobb CM. Non-surgical pocket therapy: Mechanical. Ann Periodontol. 1996; 1: 443-490.

7. Cortellini P, Pini Prato G, Tonetti MS. Periodontal regeneration of human intrabony defects. I. Clinical measures. J Periodontol 1993; 64: 254-260.

8. Döri F, Huszár T, Nikolidakis D, Tihanyi D, Horváth A, Arweiler NB, Gera I, Sculean A. Effect of Platelet-Rich Plasma on the Healing of Intrabony Defects Treated With Beta Tricalcium Phosphate and Expanded Polytetrafluoroethylene Membranes. J Periodontol 2008; 79(4): 660-669.

9. Dřizhal I, Červinka M, Taha M, Strnad L. Ŕizená tkáňová regenerace - uplatnění v parodontologii. Quintessenz - Parodontologie 2001; 2(11): 18-25.

10. Fassmann A. Ŕizená tkáňová a kostní regenerace ve stomatologii. Praha: Grada Publishing, 2002: 13-14.

11. Garrett S. Periodontal regeneration around natural teeth. Ann Periodontol (World Workshop in Periodontics) 1996; 1:621-666

12. Garrett S, Bogle G. Periodontal regeneration with bone grafts. Curr Opin Periodontol 1994: 168-177.

13. Greenstein G. Nonsurgical periodontal therapy in 2000: a literature review. J Am Dent Assoc. 2000; 131: 1580-1592.

14. Greenstein G. Periodontal response to mechanical nonsurgical therapy: a review. J Periodontol. 1992; 63: 118-130.

15. Haghighati F, Saaveh G. Essentials in periodontal regeneration. JDT 2007; 4: 78-86.

16. Harris RJ. Clinical evaluation of a composite bone graft with a calcium sulfate barrier. J Periodontol 2004; 75(50): 685-692.

17. Holinger JO, Brekke J. Role of bone substitutes, Clin Orthoped 1996; 324: 55-65.

18. Jepsen S, Eberhard J, Herrera D, Needleman I. A systematic review of guided tissue regeneration for periodontal furcation defects. What is the effect of guided tissue regeneration compared with surgical debridement in the treatment of furcation defects? J Clin Periodontol 2002; 29(3): 103-116.

19. Keagle JG, Garnick JJ, Searle JR, King GE, Morse PK. Gingival resistance to probing forces. I. Determination of optimal probe diameter. J Periodontol 1989; 60: $167-171$

20. Murphy KG, Gunsolley JC. Guided tissue regeneration for the treatment of periodontal intrabony and furcation defects. A systematic review. Ann Periodontol 2003; 8(1): 266-302.

21. Needleman I, Worthington HV, Giedrys-Leeper E, Tucker R. Guided tissue regeneration for periodontal infra-bony defects. Cochrane Database of Systematic Reviews 2006; 2: Art. No.: CD001724.

22. Paolantonio M et al. Surgical treatment of periodontal intrabony defects with calcium sulfate implant and barrier versus collagen barrier or open flap debridement alone: A 12-month randomized controlled clinical trial. J Periodontol 2008; 79(10): 1886-1893.

23. Pecora G, Andreana S, Margarone J, Covani U, Sottosanti J. Bone regeneration with a calcium sulfate barrier. Oral Surg Oral Med Oral Path Oral Radio Endo 1994; 84: 424-429. 
24. Podaropoulos L, Veis AA, Papadimitriou S, Alexandridis C, Kalyvas D. Bone regeneration using beta-tricalcium phosphate in a calcium sulfate matrix. J Ora Implantol 2009; 35(1): 28-36

25. Reddy MS, Jeffcoat MK. Methods of assessing periodontal regeneration. Periodontol 2000 1999; 19: 87-103.

26. Robinson PJ, Vitek RM. The relationship between gingival inflammation and resistance to probe penetration. J Periodontol Res 1979; 14: 239-243.

27. Rosen PS, Reynolds MA, Bowers GM. The treatment of intrabony defects with bone grafts. Periodontology 2000 2000; 22: 88-103.
28. Sukumar S, Drrízhal I. Bone grafts in periodontal therapy. Acta medica (Hradec Králové) 2008; 51(4): 203-207.

29. Tözüm TF, Erdal C, Saygun I. Treatment of Periapical Dental Implant Pathology with Guided Bone Regeneration- Case Report. Turk J Med Sci 2006; 36(3): 191-196.

30. Tözüm TF, Sencimen M, Ortakoglu K, Özdemir A, Aydin Ö, Keles M. Diagnosis and treatment of a large periapical implant lesion associated with adjacent natural tooth: a case report. Oral Surg Oral Med Oral Pathol Oral Radiol Endod 2006 Jun; 101(6): e132-138.

Received: 22/12/2009.

Accepted in revised form: 20/10/2010.

\section{Corresponding author:}

Sujith Sukumar B.D.S., University Hospital Hradec Králové, Department of Dentistry, Sokolská 581, 50005 Hradec Králové, Czech Republic; e-mail: docsujith@gmail.com 